\title{
Intestinal parasitic infections among under-five children and maternal awareness about the infections in Shesha Kekele, Wondo Genet, Southern Ethiopia
}

\author{
Liza A. Nyantekyi ${ }^{1}$, Mengistu Legesse ${ }^{2}$, Mulugeta Belay ${ }^{2}$, Konjit Tadesse $^{2}$, Kebreten Manaye $^{3}$, Chanda Macias ${ }^{3}$, \\ Berhanu Erko ${ }^{2}$
}

\begin{abstract}
Background: Few studies have reported the magnitude of intestinal parasitic infections among under-five children in tropical countries. Moreover, there is little information on maternal awareness about intestinal parasitosis.

Objective: To determine the prevalence of intestinal parasitosis among under-five children, and assess maternal awareness about it in Shesha Kebkele, Wondo Genet, Southern Ethiopia.

Methods: A cross-sectional study involving 288 under-five children was conducted and stool samples were collected and examined for intestinal parasites using Kato-Katz and formol-ether concentration methods. In addition, a total of 130 mothers of under-five children were interviewed regarding their awareness about intestinal parasitic infections.

Results: Of the 288 children, 245 (85.1\%) were found infected with one or more intestinal parasites. The prevalence of Trichuris trichiura, Schistosoma mansoni and Ascaris lumbricoides, hookworm, and Hymenolepis nana infections as determined by Kato-Katz were $74.7 \%, 37.2 \%, 25.7 \%, 5.9 \%$, and $4.5 \%$, respectively. On the other hand, the prevalence of Strongyloides stercoralis, Giardia lamblia, Entamoeba histolytica/dispar, and Entamoeba coli infections as determined by formol-ether concentration method were $0.69 \%, 13.2 \%, 0.35 \%$, and $2.1 \%$, respectively. Most mothers were reasonably aware of the mode of transmission of ascariasis, amoebiasis and giardiasis while they had very limited knowledge of bilharzia and hookworm transmission. Almost all of the respondents reported that infections with intestinal parasites could cause retardation of growth and death in children unless treated.

Conclusion: Intestinal parasitic infections were prevalent in varying magnitude among under-five children in Wondo Genet area, Southern Ethiopia. Mothers in the study area had a fairly good knowledge of the impact of infections but limited knowledge of the mode of transmission of intestinal parasitic infections. Improvement of sanitation and health education are required besides preventive chemotherapy to control worms (except for schistosomiasis in under-five which need treatment on an individual basis) and other intestinal parasitic infections in the area. (Ethiop. J. Health Dev. 2010;24(3):185-190)
\end{abstract}

\section{Introduction}

Intestinal parasitic infections are among the major public health and socio-economic concerns that adversely affect the well-being of the poor in developing countries. It has been estimated that Ascaris lumbricoides, hookworm and Trichuris trichiura infect 1,450 million, 1,300 million and 1,050 million people worldwide, respectively, while schistosomiasis affects over 200 million people (1). Entamoeba histolytica and Giardia lamblia are also estimated to infect about 60 million and 200 million people worldwide, respectively (2).

In children, intestinal parasitic infections, particularly soil-transmitted helminthiasis is the cause of common health problems in tropical countries. Younger children are predisposed to heavy infections with intestinal parasites since their immune systems are not yet fully developed (3), and they also habitually play in faecally contaminated soil. In addition to considerable mortality and morbidity, infection with intestinal helminths has been found to profoundly affect a child's mental development, growth and physical fitness while also predisposing children to other infectious agents (4-8).

Several factors like climatic conditions, poor sanitation, unsafe drinking water, and lack of toilet facilities are the main contributors to the high prevalence of intestinal parasites in the tropical and sub-tropical countries (9). Further, lack of awareness about mode of transmission of parasitic infections increases the risk of infection. Hence, a better understanding of the above factors, as well as how social, cultural, behavioral and community awareness affect the epidemiology and control of intestinal parasites may help to design effective control strategies of these diseases $(10,11)$.

Most of the previous studies conducted in Ethiopia have focused on the prevalence and distribution of intestinal parasitic infections mainly among school children (12 16). Only few studies have reported the magnitude of intestinal parasitic infections among under-five children (17-19). Furthermore, there is limited information on the

\footnotetext{
${ }^{1}$ University of California, Irvine 364 Med Surge II, Irvine, CA 92697-1275 USA;

${ }^{2}$ Aklilu Lemma Institute of Pathobiology, Addis Ababa University, P.O. Box 1176 Tel.: +251 112763091; Fax: 251

112755296; Email: berhanue@yahoo.com Addis Ababa, Ethiopia;

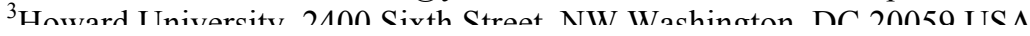


basic awareness of communities about the cause, transmission, and infection prevention in Ethiopia. Therefore, this study was designed to assess the prevalence of intestinal parasitic infections among underfive children, as well as the level of mothers' awareness about the cause, effect, mode of transmission, and preventive methods of intestinal parasitic infections in a village in Shesha Kekele, Wondo Genet, in Southern Ethiopia.

\section{Methods}

Study area and population: In June 2007, a crosssectional study was carried out on the prevalence of intestinal parasitic infections among children aged 6 months to five years and maternal awareness about intestinal parasites. Shesha Kekele is located $270 \mathrm{~km}$ south of Addis Ababa in Wondo Genet at an altitude of about $1800 \mathrm{~m}$ above sea level. Sidama, Hadiya, Wolayita and Oromo ethnic groups are the main inhabitants in the area. Most of the inhabitants earn their living as farmers practicing settled mixed agriculture. Enset (Ensete ventricosum) and maize are the principal food crops. Sugar cane and chat (Catha edulis) are the principal cash crops produced using traditional irrigation methods. Soiltransmitted helminthiasis and intestinal schistosomiasis are known to be highly prevalent in the area (15). The study population included all mothers who had under-five children. Accordingly, 130 mothers and 288 children (6 months to 5 years) found in the area in June 2007, participated in the study.

Stool sample collection and examination: Before collecting the stool samples, the aim of the study was explained to the leaders of the peasant association and permission was obtained. Next, mothers were informed to bring their under-five children (6 months to 5 years) to a central place for examination of intestinal parasitic infections. After explaining the aim of the study to the mothers, a clean piece of plastic sheet was distributed to each volunteer mother and instructed to provide about $2 \mathrm{~g}$ of fresh stool sample from their. A portion of the sample was processed by Kato method using a template delivering a plug of $41.7 \mathrm{mg}$ of stool (20) and the remaining was placed in vials containing $10 \%$ formalin. Samples processed by Kato were qualitatively examined on the spot for hookworm ova and other intestinal helminthic infections. Quantitative examination of the Kato-Katz slides for helminthiasis (except for hookworms) was done in the laboratory within one week of stool collection. Stool specimens placed in vials were also qualitatively examined in the laboratory for strongyloidiasis and protozoan parasites by the formolether concentration method.

Assessment of maternal awareness: Volunteer mothers who brought their children for examination were interviewed about the source of intestinal parasitic infection, mode of transmission, symptoms, and the effects of helminth infection on the children's health and preventive methods of some of the common intestinal parasites in the area. Each mother was interviewed in local language using open-ended questions by data the collectors selected from the study areas under the supervision of the research team.

Data analysis: The prevalence of parasitic infections was expressed in percentages and the classes of intensity of infection as light, moderate and heavy based on the egg count per gram of stool (1). The level of maternal awareness was expressed as percentage for different categories of determinants and presented in table.

Ethical considerations: The study obtained ethical clearance from the Ethical Clearance Committee of the Aklilu Lemma Institute of Pathobiology. At the end of the study, infected children were appropriately treated, while mothers were given health education about the transmission of intestinal parasites, the symptoms of infection and how to minimize and prevent infection of their children.

\section{Results}

Prevalence of intestinal parasitic infections: A total of 288 children [(148 (51.3\%) females and $140(48.6 .0 \%)$ males] aged 6 to 5 months (median age: 45 months) were examined for intestinal parasitic infections (data not shown). Of those, 245 (85.1\%) children were found to be infected with one or more intestinal parasites. The prevalence of T. trichiura, S. mansoni, A. lumbricoides, Hymenolepis nana, and hookworm infections as determined by Kato-Katz method were $74.7 \%$, 37.2\%, $25.7 \%, 4.5 \%$, and $5.9 \%$, respectively (Table 1). The prevalence of Strongyloides stercoralis, Giardia lamblia, Entamoeba histolytica/dispar and Entamoeba coli infections among children as determined by formol-ether concentration method were $13.2 \%, 0.35 \%$, and $2.1 \%$, respectively.

Table 1 presents the percentage of children infected with soil-transmitted helminths. Generally, the prevalence of infections with intestinal parasites tended to increase with age.

Of the 245 infected children, $34.5 \%, 33.3 \%$ and $23.2 \%$ had single, double and multiple parasitic infections, respectively (data not shown). The majority of the children were found to harbor light to moderate infections for $T$. trichiura, $S$. mansoni, and $A$. lumbricoides (Table 2). Although examination for hookworm was done by Kato-Katz method within one hour of preparation, classes of intensity were not determined, but noted as only the presence or absence of infection. 
Table 1: Prevalence of intestinal parasitic infections by age groups among children in Shesha Kekele Village, Wondo Genet, Southern Ethiopia, June 2007

\begin{tabular}{|c|c|c|c|c|c|}
\hline \multirow{2}{*}{ Parasite species } & \multicolumn{5}{|c|}{ Age group (in years) } \\
\hline & $\leq 1(n=13)$ & $\leq 2(n=43)$ & $\leq 3(n=53)$ & $\leq 4(n=74)$ & $\leq 5(n=105)$ \\
\hline T. trichiura & $2(15.4 \%)$ & $28(65.1 \%)$ & $41(77.4 \%)$ & $59(80.0 \%)$ & $85(81.0 \%)$ \\
\hline S. mansoni & $0(0 \%)$ & $6(14.0 \%)$ & $12(2.6 \%)$ & $33(45.0 \%)$ & $56(53.3 \%)$ \\
\hline A. lumbricoides & $1(7.7 \%)$ & $6(14.0 \%)$ & $16(30.2 \%)$ & $16(21.6 \%)$ & $35(38.7 \%)$ \\
\hline H. nana & $0(0 \%)$ & $0(7.0 \%)$ & $11.9 \%)$ & $4(5.4 \%)$ & $8(7.6 \%)$ \\
\hline Hookworms & $0(0 \%)$ & $4(9.3 \%)$ & $3(5.7 \%)$ & $4(5.4 \%)$ & $6(5.7 \%)$ \\
\hline G. lamblia** & $2(14.3 \%)$ & $11(25.6 \%)$ & $12(22.2 \%)$ & $22(29.3 \%)$ & $20(18.9 \%)$ \\
\hline Others ${ }^{* *}$ & $0(0 \%)$ & $1(2.3 \%)$ & $2(3.8 \%)$ & $3(4.1 \%)$ & $8(7.6 \%)$ \\
\hline
\end{tabular}

** Examined by formol-ether concentration (FEC).

** Others examined by FEC include S. stercoralis, E.histolytica/dispar and E. coli.

Table 2: Intensity of helminthic infections among under-five children in Shesha Kekele Village, Wondo Genet, Southern Ethiopia, June 2007

\begin{tabular}{llll}
\hline & \multicolumn{3}{c}{ Intensity of infections } \\
\cline { 2 - 4 } Helminth species & Light & Moderate & Heavy \\
\hline S. mansoni & $53 / 102(52.0 \%)$ & $34 / 102(33.3 \%)$ & $15 / 102(14.7 \%)$ \\
A. lumbricoides & $30 / 65(46.2 \%)$ & $31 / 65(47.7 \%)$ & $4 / 65(6.2 \%)$ \\
T. trichiura & $170 / 205(82.9 \%)$ & $31 / 205(15.1 \%)$ & $2 / 205(1.0 \%)$ \\
\hline
\end{tabular}

Table 3: Knowledge of mothers about intestinal parasitic infections in Shesha Kekele Village, Wondo Genet, Southern Ethiopia, June 2007

\begin{tabular}{|c|c|}
\hline Variables & Number of respondents $(\%), n=130$ \\
\hline \multicolumn{2}{|l|}{ Do you know how a child gets infection with intestinal parasites? } \\
\hline Yes & $116(89.2 \%)$ \\
\hline No & $14(10.8 \%)$ \\
\hline \multicolumn{2}{|l|}{ How do children get ascariasis? } \\
\hline Chewing sugar cane & $45(38.8 \%)$ \\
\hline Eating contaminated food & $27(23.3 \%)$ \\
\hline Drinking dirty water & $23(19.8 \%)$ \\
\hline Eating enset, uncooked cabbage & $23(19.8 \%)$ \\
\hline Eating sweet food, potato, raw milk, evil eye & $42(36.2 \%)$ \\
\hline Do not know & $12(10.3 \%)$ \\
\hline \multicolumn{2}{|l|}{ How do children get hookworm? } \\
\hline Drinking dirty water & $6(5.2 \%)$ \\
\hline Other & $4(3.4 \%)$ \\
\hline Do not know & $110(94.8 \%)$ \\
\hline \multicolumn{2}{|l|}{ How do children get bilharzia (schistosomiasis)? } \\
\hline Drinking dirty/river water & $19(16.4 \%)$ \\
\hline Washing in river water & $8(6.9 \%)$ \\
\hline Others (bad air, contaminated food, poor sanitation) & $13(11.2 \%)$ \\
\hline Do not know & $82(70.7 \%)$ \\
\hline \multicolumn{2}{|l|}{ How do children get amoebiasis? } \\
\hline Eating uncooked cabbage, red/green pepper & $45(38.8 \%)$ \\
\hline Drinking dirty water & $22(19.0 \%)$ \\
\hline Eating contaminated food & $10(8.6 \%)$ \\
\hline Others like eating tomato, potato, sugar cane and lack of food & $42(36.2 \%)$ \\
\hline Do not know & $21(18.1 \%)$ \\
\hline \multicolumn{2}{|l|}{ How do children get giardiasis? } \\
\hline Drinking dirty/river water & $67(57.8 \%)$ \\
\hline Others like contaminated food, lack of food and poor sanitation & $19(16.3 \%)$ \\
\hline Do not know & $37(31.9 \%)$ \\
\hline
\end{tabular}

Maternal awareness: A total of 130 mothers (aged 1860 years, mean age 29.7) were interviewed about intestinal parasitic infections. The majority of the participants were farmers $(86.9 \%)$, Christians $(91.5 \%)$ and illiterate $(55.4 \%)$. Ethnicity wise, 30.8\%, 24.6\%, $13.1 \%$ and $31.5 \%$ of the participants were from Oromo, Sidama, Wolayta and others, respectively.
Results of the questionnaire survey showed that the majority of the mothers were reasonably aware about transmission of ascariasis, amoebiasis and giardiasis (Table 3). When asked specific questions such as how a child gets ascariasis, $38.8 \%$ of the mothers associated the cause with eating sugar cane, while others mentioned various causes like eating contaminated food, drinking Fthisn I Hostth Dow, on1n.ou(z) 
dirty water, and eating uncooked cabbage. Consumption of uncooked cabbage or red/green pepper was suggested by the mothers $(38.8 \%)$ as a major cause of amoebiasis in children, though others mentioned drinking dirty water, eating contaminated food or consuming tomato and potato as the cause of amoebiasis. Most of the mothers $(57.8 \%)$ responded that unclean water is the cause of giardiasis. $70.7 \%$ and $94.8 \%$ of the mothers responded that they do not know how children get bilharzia and hookworm infections, respectively.

Regarding the symptoms of intestinal parasitic infections, almost all the mothers suggested one or more symptoms like diarrhea $(50.8 \%)$, vomiting $(39.2 \%)$, loss of appetite (36.9\%), abdominal discomfort (35.3\%) and an enlarged abdomen (30\%). They also responded that infection with intestinal parasites could cause growth retardation, thinness, weakness, and child death.

\section{Discussion}

In this study, the prevalence of both intestinal helminthic and protozoan infections among children aged 6 months to five years was determined in Shesha Kekele Village in Wondo Genet, Southern Ethiopia. The results of the study revealed the presence of various intestinal parasitic infections in varying degrees among under-five children. Among helminthiasis, trichuriasis was the most prevalent infection, followed by intestinal schistosomiasis and ascariasis, whereas giardiasis was the leading infection among protozoan infections. The high prevalence of trichuriasis observed in this study was comparable to the results of previous community-based studies in the same area (15). However, in contrast to a previous study conducted in other intestinal schistosomiasis endemic areas of Ethiopia (18), the present study revealed high prevalence of Schistosoma mansoni infection among under-five children. The trend of infections with the different parasites was found to increase with age, in agreement with the observation among under-five children in Kenya (21).

Heavy infections with T. trichiura, Schistosoma mansoni, $A$. lumbricoides and $G$. lamblia are known to affect childhood health. Previous studies have revealed that moderate to heavy infection with $T$. trichiura could result in chronic dysentery commonly known as Trichuris dysentery syndrome (TDS), rectal prolapse, iron deficiency anaemia, growth and mental impairment (22 24). Similarly, ascariasis is associated with severe morbidity such as intestinal obstruction. Biliary and pancreatic ascariasis can result in mortality, reduced physical growth and cognitive development depending upon the intensity of infection (5). Evidence has also indicated that infection with $G$. lamblia could cause malabsorption, chronic diarrhea and long-term growth retardation in children (25). Intestinal schistosomiasis could cause diarrhea, loss of appetite, loss of weight, growth retardation, cognitive defects and hepatosplenomegaly in chronic cases which may lead to death in children $(26,27)$. Although the impact of the infections on the children was not assessed in this study, it is likely that the infections would exert considerable health impact on the children depending on the intensity of the infection.

High prevalence of trichuriasis (74.7\%) and S. mansoni (37.2\%) observed among under-five children are a serious concern because health problem in this age group is compounded by co-infection of these worms with malaria. In addition, since there is no documented information on the safety of antischistosomal drug (praziquantel) for children under 4 years of age at the moment, preventive chemotherapy for schistosomiasis is not indicated for this age group (28). Nevetheless, children of this age group can be treated on an individual basis by medical personnel.

Except for bilharzia and hookworm infections, questionnaire survey results indicated that mothers have some knowledge about the modes of transmission of intestinal parasites. $70.7 \%$ and $94.8 \%$ of the mothers responded that they do not know how children get bilharzia and hookworm infections, respectively. This is also partly attributed to failure of the study to identify and use appropriate terms by which these parasites are known locally, particularly for hookworm.

Mothers had relatively reasonable knowledge about intestinal parasitic infections, and they are very well aware of their impact. In agreement with the study from Upper Egypt (29), almost all of the mothers/respondents realized that infection with intestinal parasites could cause serious health problems including growth retardation, malnutrition and child death unless treated.

Availability of effective drugs, community awareness about the etiologic agents, and mode of transmission of intestinal parasites could contribute to the development of integrated control strategies of parasitic infections (29 - 31). In this study, we assessed mothers' awareness about the cause of infection, mode of transmission, symptoms, impact of the infection on children's health, and methods of prevention of common intestinal parasitic infections. Unlike studies in Nigeria (32), the present observations showed that mothers in the study area had some knowledge about mode of transmission of ascariasis, giardiasis, and amoebiasis. Most of the mothers responded that drinking river water, chewing sugar cane, feeding a child with uncooked cabbage and green pepper are responsible for ascariasis and amoebiasis. Observation also showed that chewing sugar cane is a common practice among children in the area and it is possible that children get infection from sugar cane contaminated with parasite agents. Obviously, consumption of uncooked contaminated vegetables also serves as a means of intestinal parasitic infections. 
In conclusion, the results of the present study revealed that intestinal parasitic infections were prevalent in varying magnitude among under-five children in the study area. The study also revealed that mothers in the study area had limited or some knowledge about various intestinal parasitic infections, and they were aware of the impact. In addition to preventive chemotherapy for worms, improvement of sanitation and provision of health education are required to control and eliminate all intestinal parasitic infections in the area.

\section{Acknowledgments}

The authors would like to acknowledge community leaders of the study area and the study participants for their cooperation during the survey. We are also grateful to the technical staff of the Medical Parasitology Unit of the Aklilu Lemma Institute of Pathobiology, Addis Ababa University, for their technical assistance. This work was financially supported by Howard University's, MHIRT Program (NIH/MHIRT 9T37 MD001582-08).

\section{References}

1. WHO Expert Committee. Prevention and control of schistosomiasis and soil-transmitted helminthiases. WHO Technical Report Series, 2002;912:1-57.

2. Murray PR, Rosenthal KS, Kobayashi G.S, Pfalle HA. Medical Microbiology. $4^{\text {th }}$ ed. London: Mosby; 2002: 681-761.

3. Rao V, Sugunan A, Murhekar M, Sehgal S. Malnutrition and high childhood mortality among the Onge tribe of the Andaman and Nicobar Islands. Public Health Nutrition 2006;9:19-25.

4. Stephenson L, Latham, Adams E, Kinoti S, Pertet A. Weight gain of Kenyan school children infected with hookworm, Trichuris trichiura and Ascaris lumbricoides is improved following once-or twiceyearly treatment with albendazole. Journal of Nutrition 1993;123:656-665.

5. Simeon DT, Grantham-McGregor SM, Callender JE, Wong MS. Treatment of Trichuris trichiura infections improves growth, spelling scores and school attendance in some children. Journal of Nutrition 1995;125:1875-1883.

6. De Silva NR, Chan MS, Bundy DAP. Morbidity and mortality due to ascariasis: re-estimation and sensitivity analysis of global numbers at risk. Tropical Medicine and International Health 1997;2:519-28.

7. Albonico M, Crompton DWT, Savioli L. Control strategies for human intestinal nematode infections. Advances in Parasitology 1998;42:277-341.

8. Elias D, Wolday D, Akuffo H, Petros B, Bronner $\mathrm{U}$, Britton S. Effect of deworming on human T cell responses to mycobacterium antigen in helminth exposed individuals before and after BCG vaccination. Clinical Experimental Immunology 2001;123:219-25.

9. Mahfouz AAR, El-Morshedy H, Farghaly A, Khalil A. Ecological determinants of intestinal parasitic infections among pre-school children in an Urban
Squatter Settlement of Egypt. Journal of Tropical Pediatric 1997;43:341-44.

10. Kloos H. Human behavior, health education and schistosomiasis control: A review. Soc Sci Med 1995;40:1497-1511.

11. Pearce N. Traditional epidemiology, modern epidemiology and public health. American Journal of Public Health 1996;86:678-83.

12. McConnell E, Armstrong JC. Intestinal parasitism in fifty communities on the central plateau of Ethiopia. Ethiop Med J 1976;14:159-168.

13. Tedla S. Hookworm infections in several communities in Ethiopia. East African Medical Journal 1986;63:134-39.

14. Assefa T, Wolde Micheal T, Dejene A. Intestinal parasitism among students in three localities in south Wello, Ethiopia. Ethiop J Health Dev 1998;12:231235.

15. Erko B, Medhin G. Human helminthiasis in Wondo Genet, Southern Ethiopia, with emphasis on geohelminthiasis. Ethiop Med J 2003;41:333-44.

16. Legesse M, Erko B. Prevalence of intestinal parasites among schoolchildren in a rural area close to the southeast of Lake Langano. Ethiopia. Ethiop $J$ Health Dev 2004;18:116-20.

17. Taticheff S, Abdulahi Y, Haile Meskal F. Intestinal parasitic infection in pre-school children in Addis Ababa. Ethiop Med J 1981;19:35-40.

18. Birrie H, Balcha F, Abebe F. Intestinal parasitosis among under-fives in two communities in Ethiopia. Ethiop J Health Dev 1998;12:63-67.

19. Asfaw S, Goitom L. Malnutrition and enteric parasitoses among under-five children in Analem Village, Tigray. Ethiop J Health Dev 2000;14:6775.

20. WHO. Basic Laboratory Methods in Medical Parasitology. Geneva: WHO; 1991.

21. Brooker S, Peshu N, Warn PA, Mosobo M, Guyatt HL, Marsh K, Snow RW. The epidemiology of hookworm infection and its contribution to anaemia among pre-school children on the Kenyan coast. Transaction of the Royal Society of Tropical Medicine and Hygiene 1999;93:240-46.

22. Bundy DAP, Cooper ES. Trichuris and trichuriasis in humans. Advances in Parasitology 1989; 28: 107-173.

23. Cooper ES, Bundy DAP, MacDonald TT, Golden MHN. Growth suppression in the Trichuris dysentery syndrome. European Journal of Clinical Nutrition 1990; 44: 285-291.

24. Ramdath DD, Simeon DT, Wong MS, GranthamMcGregon SM. Iron status of schoolchildren with varying intensities of Trichuris trichiura infection. Parasitology 1994; 110: 347-51.

25. Fraser DN, Bilenko N, Deckelbaum RJ, Dagan R, El-On J, Naggan L. Giardia lamblia carriage in Israeli Bedouin infants: risk factors and consequences. Clinical Infectious Diseases 2000; 30: 419-424. 
26. McGarvey ST, Wu G, Zhang S, Wang Y, Peters P, Olds GR, Wiest PM. Child growth, nutritional status and Schistosomiasis japonica in Jiangxi, People's Republic of China. American Journal of Tropical Medicine and Hygiene 1993; 48: 547-553.

27. Ross AGP, Sleigh AC, Li Y, Davis GM, Williams GM, Jiang Z, Feng Z, McManus P. Schistosomiasis in the people's Republic of China: prospects and challenges for the $21^{\text {st }}$ Century. Clinical Microbiology Review 2001;14:270-295.

28. World Health Organization. Preventive chemotherapy in human helminthiasis: coordinated use of anthelminthic drugs in control interventions: A manual for health professionals and program managers. Geneva: WHO; 2006.

29. Curtale F, Pezzotti P, Sharbini A, Maadat H, Ingrosso P, Saad Y, Babille M. Knowledge, perceptions and behavior of mothers toward intestinal helminthes in Upper Egypt: implications for control. Health Policy and Planning 1998;13:423-32.

30. Asaolu SO, Ofoezie IE. The role of health education and sanitation in the control of helminth infections. Acta Tropica 2003;86:283-94.

31. Quihui L, Valencia ME, Crompton DWT, Phillips S, Hagan P, Morales G, Diaz-Camacho SP. Role of the employment status and education of mothers in the prevalence of intestinal parasitic infections in Mexican rural schoolchildren. BMC Public Health 2006;6:225-36.

32. Omoigberale A, Airauhi L. Aspects of the epidemiology of intestinal parasitoses (IP) in children: knowledge, practices and perceptions of mothers. Nigerian Journal of Clinical Practice 2006;9:109-13. 